\title{
Expertengespräch: Urogenitalerkrankungen - Teil 1
}

Ob schmerzende Harnröhre, Brennen der Schamlippen oder Blasenbeschwerden - Frauen seien gut beraten, urogenitale Beschwerden umfassend anzugehen, sagt die Frauenärztin Dr. med. Regina Widmer. Auch die Selbstkompetenz gehöre dazu.

Frau Dr. Widmer, was unterscheidet Sie von einer ausschliesslich schulmedizinisch tätigen Frauenärztin?

Widmer: Ich habe im Medizinstudium, also vor gut 30 Jahren, begonnen, mich mit Komplementärmedizin zu beschäftigen. Damals war die Zeit der Frauengesundheitsbewegung, und seither hat mich das Gebiet nie mehr losgelassen. Heute biete ich beides an, sowohl schulmedizinische Gynäkologie als auch Komplementärmedizin. Meine Patientinnen können auswählen. Dabei stütze ich mich vor allem auf die Phytotherapie, aber ich «bediene» mich auch anderer Methoden - z.B. solcher der anthroposophischen Medizin, der Homöopathie oder der Akupunktur.

\section{Welche Frauen kommen zu Ihnen?}

Zum Teil suchen sie einfach eine Frauenärztin. Unsere Praxis ist aus der Oberärztinnen-Sprechstunde des Bürgerspitals Solothurn entstanden; von daher haben wir die ganz normale Klientel. Mit den Jahren begannen uns vermehrt Frauen aufzusuchen, die gehört hatten, dass wir uns mehr Zeit nehmen und grundsätzlich mehr anbieten können als die Schulmedizin. Wir haben auch unsere Spezialitäten, insbesondere Psychosomatik, urogenitale Beschwerden und Sexualmedizin.
Treffen Sie urogenitale Erkrankungen im Praxisalltag häufig an?

Ja. Seit ich zu diesem Thema publiziert habe, bekomme ich regelmässig Zuweisungen mit zum Teil sehr komplexen Situationen. Beispielsweise Vulvodynien, bei denen die Schulmedizinerin oft nicht weiterkommt. In vielen Fällen ist unklar, ob es nun die Harnröhre oder die Vulva ist, die Probleme bereitet. Der Verlauf solcher Geschichten ist oft chronisch, mit schmerzendem Geschlechtsverkehr und anderen Unannehmlichkeiten. Um solche Situationen kümmere ich mich mit grossem Ehrgeiz. Akute Befunde wie Harnwegsinfekte sind problemlos. Diese behandle ich entweder antibiotisch oder mit anthroposophischen und/oder phytotherapeutischen Mitteln. Auch Urethralbeschwerden und Reizblasen heute bezeichnet man sie eher als «Overactive Bladder» - sehe ich oft.

\section{Wie behandeln Sie solche Beschwerden?}

Akupunktur und Moxa biete ich bei chronischen Urethra- und Blasenbeschwerden konsequent an. Erst wird über der Symphyse genadelt, dann ein Stück einer Moxa-Zigarre, also chinesischem Beifuss, aufgesetzt, das abgebrannt wird. So gelangt Wärme tief ins System, oft bis in die Urethra. Das ist sehr einfach und wirksam. Um das zu lernen, braucht es nicht einmal eine langjährige TCM-Ausbildung. Beim Urethralsyndrom ist eine lokale Wärmeapplikation an der Vulva hilfreich.

Was tun Sie als Erstes, wenn eine Frau Sie mit chronischen Harnwegsinfekten konsultiert?

Als Erstes reserviere ich mir viel Zeit - bei der ersten Konsultation wenn möglich $45 \mathrm{~min}$. Dann heisst es, eine genaue Anamnese zu machen: Wann genau hat alles zum ersten Mal und wie begonnen? Bei chronischen Verläufen ist immer das Sexualleben in Mitleidenschaft gezogen. Oft leiden Frauen sogar mehr unter den Auswirkungen auf das Intimleben als unter den organischen Beschwerden. Das ist immer noch ein Tabu, auch für die Ärzte. Es ist wichtig, das Thema schon in der Erstkonsultation offen anzusprechen. Am Ende gebe ich der Patientin etwas Konkretes mit auf den Weg, z.B. ein Wärmepflaster oder Tipps zur Intimpflege - für mich das A und O. Nach ein paar Wochen sehe ich sie ein zweites Mal; ich lade sie immer wieder ein, bis sie mich nicht mehr braucht. Verblüffend dabei: Es kommt manchmal weniger auf die medizinischen Handlungen an als auf die Zuwendung, darauf, dass die Frauen wieder daran glauben können, dass ihre schmerzende Vulva, 


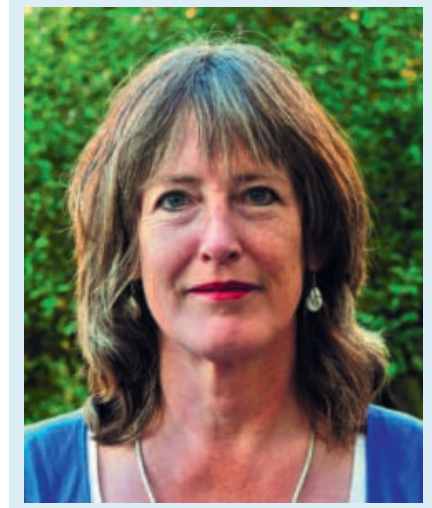

Dr. med. Regina Widmer

ist Frauenärztin FMH und Co-Geschäftsleiterin der Gemeinschaftspraxis Runa in Solothurn. In der Frauenpraxis Runa werden Gynäkologie und Geburtshilfe angeboten, basierend auf der Gleichwertigkeit von Schul- und Komplementärmedizin. Frau Dr. Widmers komplementärmedizinische Schwerpunkte sind Phytotherapie und Akupunktur bei Harnwegsbeschwerden sowie Sexualtherapie. Sie ist Koautorin des kürzlich erschienenen Buchs «Wechseljahre - natürlich begleitet» (BeobachterBuchverlag, 2011).

«Viele Ärztinnen und Ärzte scheuen sich davor, genau nachzufragen, was den Frauen im Genitalbereich wehtut. Nicht nachzufragen ist aber eine Unterlassung.»

Harnröhre oder Blase wieder gesund werden kann. Das ist eine wesentliche Voraussetzung für eine Heilung.

\section{Wie wichtig sind die psychischen Faktoren?}

Psychische Faktoren spielen eine grosse Rolle, gerade beim Unterleib. Das Selbstbild, die Interaktion mit dem Partner oder Leistungsdruck sind häufige Themen. Natürlich will man dem Körper etwas geben, aber wir brauchen mehr auf verschiedenen Ebenen, um heil zu werden. So kann es gelingen, jemanden von einem chronischen Leiden wegzuführen oder zumindest dabei zu unterstützen, besser damit umzugehen.
Sie schreiben in einem Fachartikel, das postkoitale Urethralsyndrom werde oft verkannt oder als Blasenschwäche fehlgedeutet und tabuisiert. Gibt es bei den urogenitalen Erkrankungen noch weitere Tabus?

Ja sicher. In der Medizin wird oft nicht zwischen Blase und Harnröhre, der Urethra, unterschieden, dabei ist die Differenzierung meist einfach: Schmerzt es bei der Miktion oberhalb der Symphyse, ist es die Blase, unterhalb ist es die Harnröhre. Werden die Frauen während der Anamnese aufgefordert, mit der Hand zu zeigen, wo es wehtut, ist es sonnenklar: Die Hand über der Symphyse weist auf die Blase hin, die Hand auf dem Mons Pubis oder der Vulva auf die Harnröhre. So einfach ist es oft. Weil es die Sexualität tangiert, scheuen sich viele Ärzte und auch Ärztinnen nachzufragen, was genau im Genitalbereich wehtut, wenn es brennt beim Wasserlösen. Nicht nachzufragen ist aber eine Unterlassung. Nachzufragen ist Ausdruck einer professionellen Neugier, die wir entwickeln müssen. Sie ist oft der Schlüssel, Bewegung in chronifizierte Leidensgeschichten zu bringen.

Viele Frauen, ob jung oder alt, leiden unter Jucken der Scheide mit oder ohne Ausfluss. Was raten Sie in diesem Fall?

Häufigster Grund für Jucken sind Pilze und für übelriechenden Ausfluss ein bakterielles Ungleichgewicht (bakterielle Vaginose). Hier behandelt man mit allem, was ansäuert. Schmerzen und Brennen ohne ersichtlichen Grund bilden eine andere Kategorie. Oft ist es empfindliche Haut. Hier wie auch bei Pilz oder bakterieller Vaginose heisst es: Den Scheidenvorhof fetten, fetten, fetten! Die Intimpflege ist auch als Sekundärprophylaxe sehr wichtig. Die Haut muss nach der Akutbehandlung aufgebaut und in Form gebracht werden; sie darf keine Risse haben, muss elastisch und strapazierfähig sein. Für die Intimpflege gibt es wunderbare pflanzliche Mittel, z.B. hochwertige Kosmetikaprodukte. Ob eine Frau lieber ölt oder cremt, muss sie für sich selbst herausfinden.

\section{Sie haben kürzlich ein Buch über die Menopause geschrieben (siehe auch Buchbesprechung in diesem Heft). Eine Ihrer Thesen lautet, der Prämenopause werde zu wenig Beachtung geschenkt. Warum?}

Das wird in der Fachausbildung kaum gelehrt. Die Prämenopause betrifft Frauen zwischen 45 und 50; es ist die Zeit der Östrogendominanz: Bevor Frauen Wallungen bekommen, registrieren sie oft kürzere Zyklen, stärkere Blutungen und prämenstruelle Reizbarkeit, Niedergeschlagenheit und Brustspannen. Gegen die Symptome hilft Mönchspfeffer am besten - in der Form von Extrakten (Dragées).

Nehmen die urogenitalen Beschwerden nach der Menopause bei allen Frauen zu?

Nicht bei allen. Aber die Haut wird dünner; dadurch leiden viele Frauen unter Harnröhren- und Blasenreizungen, trockener Scheide und dadurch Schmerzen beim Geschlechtsverkehr. Es gibt andererseits Frauen, die bestens durchblutete Genitalien haben. Es sind eher die Ausnahmen. Den Frauen rate ich, ein Öl zu benutzen, aber auch, sexuell aktiv zu sein - dann sind die Genitalien automatisch besser durchblutet.

Ihr Buch hält eine Fülle von Pflanzenheilmitteln für solche Beschwerden bereit. Können Sie uns die wichtigsten Phytotherapeutika nennen, die 
Die drei wichtigsten Pflanzen sind der Mönchspfeffer für die prämenopausalen Beschwerden, die Traubensilberkerze bei Wallungen und das Johanniskraut bei Stimmungstiefs und Schlafstörungen. Daneben existiert ein grosser Reichtum an Pflanzen, die individuell eingesetzt werden können. Wichtig sind auch Omega-3-Fettsäuren. Ein wunderbares Produkt für Wechseljährige ist Triolinum, das neben Omega-3-Fettsäuren auf Leinsamen-Basis auch Phytoöstrogene enthält.

Welche anderen komplementärmedizinischen Methoden eignen sich für urogenitale Beschwerden in der Menopause?

Grundsätzlich: Wenn man mit der Schulmedizin an Grenzen stösst, soll man etwas anderes ausprobieren, einen anderen Zugang suchen - seien dies Kräuter, Akupunkturnadeln oder Globuli. Ich befürworte stark die Selbstmedikation und das Selbst-Ausprobieren in einem vernünftigen Rahmen. Dafür steht auch unser Buch über die natürliche Begleitung der Wechseljahre. Das Schlagwort «Fragen Sie Ihren Arzt oder Apotheker» gilt aus meiner Sicht nur bedingt. Wichtiger ist es, die Selbstkompetenz zu stärken.

Interview: Irène Dietschi 